\title{
Design and Analysis of Wideband Ladder-Type Film Bulk Acoustic Wave Resonator Filters in Ku-Band
}

\author{
N. Izza M. Nor, ${ }^{1,2}$ K. Shah, ${ }^{1}$ J. Singh, ${ }^{1}$ and Z. Sauli ${ }^{2}$ \\ ${ }^{1}$ Centre for Technology Infusion, La Trobe University, Bundoora, VIC 3086, Australia \\ ${ }^{2}$ School of Microelectronic Engineering, Universiti Malaysia Perlis, 02600 Perlis, Malaysia \\ Correspondence should be addressed to N. Izza M. Nor; nimohdnor@students.latrobe.edu.au
}

Received 10 September 2012; Accepted 3 June 2013

Academic Editor: Egidio Ragonese

Copyright (C) 2013 N. Izza M. Nor et al. This is an open access article distributed under the Creative Commons Attribution License, which permits unrestricted use, distribution, and reproduction in any medium, provided the original work is properly cited.

This paper presents the design of ladder-type filters based on film bulk acoustic wave resonator (FBAR) in Ku-band. The proposed FBAR filter has an insertion loss of $-3 \mathrm{~dB}$, out-of-band rejection of $-12 \mathrm{~dB}$ and $3 \mathrm{~dB}$ bandwidth of $1.0 \mathrm{GHz}$ from $15 \mathrm{GHz}$ to $16 \mathrm{GHz}$. Based on the characteristics of the FBAR filter, the expected characteristics of FBAR resonators are determined by using the 1D numerical analysis. This design proves that it is possible to design a wide-bandwidth FBAR filter in Ku-band.

\section{Introduction}

Transceiver systems working in Ku-band frequency range of $12-18 \mathrm{GHz}$ are primarily used for satellite communications and radars. Cost, area, and power consumption are the key figures of merit for such transceiver system. However, the literature shows that most of these transceivers are designed using low-temperature cofire ceramic (LTCC) technology and are relatively large in size and heavy due to the use of discrete components such as filter and separately located modules. The multilayer LTCC and the systems-on-package (SOP) implementations are capable of overcoming these issues by integrating active and passive components on one board. Various $\mathrm{Ku}$-band filters have been reported in the literature using different designs and manufacturing methods such as defect ground structure (DGS), interdigital structure, coupled line filters, and couple strip line filters have been integrated using LTCC technology [1-3]. However, improvement in filter performance and better integration methods with microwave monolithic integrated circuit (MMIC) and radio frequency (RF) microelectromechanical systems (MEMS) technology as used in WiFi and WiMAX applications [4] can be used to improve integration and reduce power consumption.

Film bulk acoustic wave resonator (FBAR) filter and FBAR diplexer designed using RF MEMS technology have been developed for WiFi and WiMAX applications [4]. Such MEMS components have shown better performance and higher integration level which can also be achieved in $\mathrm{Ku}-$ band transceivers using MEMS-based FBAR filters. Thin membrane-type resonator and solidly mounted resonator (SMR) are two types of FBARs based on acoustical isolation from the substrate [5]. Acoustical isolation achieved by airgap is the preferred method to achieve high-quality $(Q)$ factor using simpler fabrication methods [6]. The literature shows that AlN is the preferred material due to its moderate mechanical coupling factor, higher acoustic velocity, and higher $Q$ value [7] at operating frequencies higher than $10 \mathrm{GHz}$. Surface acoustic wave (SAW) resonators whose resonance frequency depends on the pitch of integrated device technology (IDT) electrode patterns [8] are limited to less than $15 \mathrm{GHz}$ [9]. However, FBARs operating in frequency range of $5 \mathrm{GHz}$ to $20 \mathrm{GHz}$ have been reported in, the literature $[8,10]$. Due to high $Q$ factor of FBARs, low insertion loss filters can be designed in Ku-band which will improve system performance and allow higher integration resulting in lost cost and low-power consumption [11].

There are several topologies of FBAR filters including ladder-type, lattice-type, or the combination of both [12]. FBAR filters have very high $Q$ and small size. In addition, FBAR can be manufactured on a flat silicon substrate using surface micromachining and befits mass production, and 
TABLE 1: Specifications of Ku-band transceiver front-end [3].

\begin{tabular}{|c|c|c|c|c|c|c|c|}
\hline & $\begin{array}{l}\text { Input frequency } \\
(\mathrm{GHz})\end{array}$ & $\begin{array}{l}\text { Output frequency } \\
(\mathrm{GHz})\end{array}$ & $\begin{array}{l}\text { Input power } \\
(\mathrm{dBm})\end{array}$ & $\begin{array}{l}\text { Output power } \\
(\mathrm{dBm})\end{array}$ & $\begin{array}{l}\text { Gain } \\
(\mathrm{dB})\end{array}$ & $\begin{array}{l}\mathrm{NF} \\
(\mathrm{dB})\end{array}$ & $\begin{array}{l}\text { Reject ion } \\
\quad(\mathrm{dBc})\end{array}$ \\
\hline Receiver & $15.5 \pm 0.5$ & $0.8 \pm 0.5$ & $\leq-10$ & - & $\geq 25$ & $\leq 5.5$ & - \\
\hline Transmitter & $0.8 \pm 0.5$ & $15.5 \pm 0.5$ & 0 & $\geq 27$ & $\geq 27$ & - & $\geq 35$ \\
\hline IF & \multicolumn{7}{|c|}{ LO frequency: $14.7 \mathrm{GHz}$} \\
\hline
\end{tabular}

this allows the filter components to become compact and cheap [13]. Furthermore, FBAR is compatible MMIC and MEMS technology. FBAR filter has been reported operating in frequency range of X-band, K-band, and Ka-band [13-15].

The $Q$ factor and electromechanical coupling coefficient $\left(k_{\text {eff }}^{2}\right)$ of the piezoelectric thin film are two figures of merit for an FBAR. Lower value of $k_{\text {eff }}^{2}$ leads to a high insertion loss and higher value of $Q$ leads to lower insertion loss [16]. It is important to notice that improving $k_{\text {eff }}^{2}$ results in decreased $Q$ factor, and so optimization of both parameters is defined by one figure of merit (FOM), which is a product of $k_{\mathrm{eff}}^{2} \times Q$ [16]. In filter applications, it has been shown that this FOM parameter is inversely proportional to filter insertion loss [16].

The literature shows that traditionally FBARs with high $Q$ factors are designed and used in FBAR filter circuits to evaluate their performance. The filters are manufactured and their performance is measured. However, in this research collections of closed-form expressions from [17] are used to analyse the filter performance based on FBAR design variables like series and parallel resonance frequencies, $Q$ factor, coupling coefficient along with filter design variables like filter order, insertion loss, and out-of-band rejection. A typical Ku-band transceiver specification shows that Ku-band FBAR filters are expected to have insertion loss less than $3 \mathrm{~dB}$, out-of-band rejection smaller than $12 \mathrm{~dB}$, and $3 \mathrm{~dB}$ bandwidth of $1.2 \mathrm{GHz}$ [3]. Then, based on the FBAR filter characteristics modelled using Butterworth Van Dyke (BVD) circuit [18], the expected FBAR characteristics are determined.

This paper is organised as follows: Section 2 presents the introduction of $\mathrm{Ku}$-band transceiver front-end. Section 3 describes the theory of FBAR filter design. Section 4 presents $\mathrm{Ku}$-band FBAR filter. Section 5 presents results and Section 6 presents conclusion.

\section{Ku-Band Transceiver Front-End}

A typical Ku-band transceiver consisting of low-noise amplifier (LNA), power amplifier (PA), voltage-controlled oscillator (VCO), local oscillator (LO), upconveter, downconverter, and filters is presented in Figure 1 as in [3]. In this transceiver, two types of $\mathrm{Ku}$-band filter are used which have centre frequency of $15.5 \mathrm{GHz}$ and $14.7 \mathrm{GHz}$. The filter operating at the centre frequency of $15.5 \mathrm{GHz}$ is used to select the frequency band while the filter operating at the centre frequency of $14.7 \mathrm{GHz}$ is used for filtering LO signal. The specifications of this transceiver are summarised in Table 1. Many different Ku-band transceivers exist [3, 19-21], but for

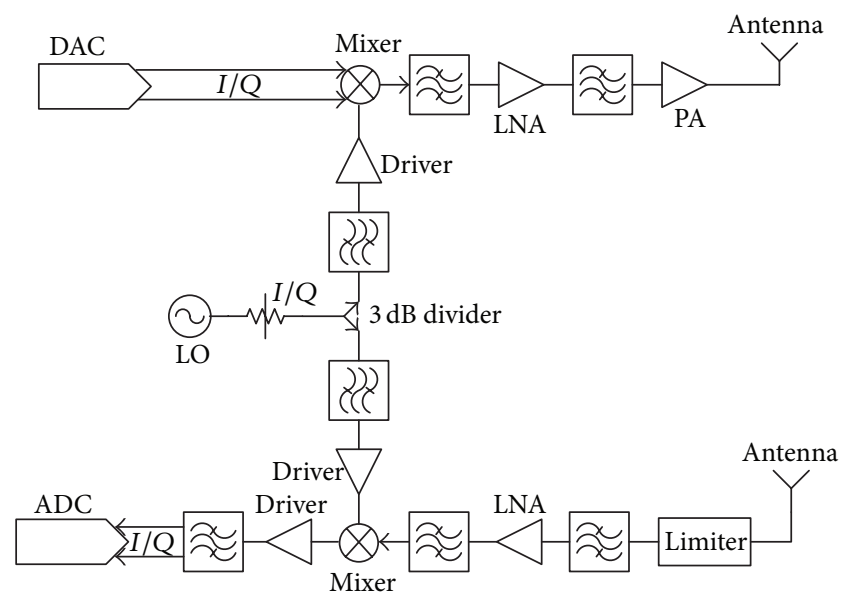

FIGURE 1: Front-end of Ku-band transceiver [3].

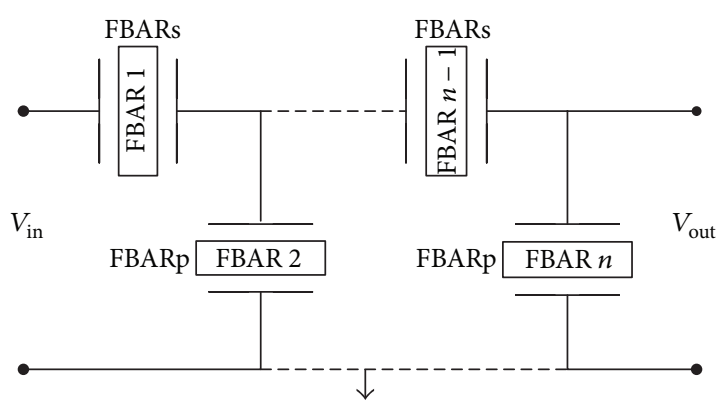

Figure 2: $n$th order of ladder filter.

this research this typical transceiver is selected to obtain performance parameters for a typical Ku-band filter working at centre frequency of $15.5 \mathrm{GHz}$. In this study, the laddertype FBAR filter is designed based on typical $\mathrm{Ku}$-band filter specifications and the characteristics of the FBAR filter and the striplines interdigital filter are compared. The next section discusses design process of FBAR filter based on closed-form equations.

\section{FBAR Filter Design}

The ladder-type FBAR filter consists of a number of Lsections comprising two resonators, one connected in series and the other connected in shunt as shown in Figure 2. The order of the filter is equal to the number of FBARs used in ladder connection. The resonator has two resonant frequencies which are series resonant frequency, $f_{s}$, and parallel resonant 


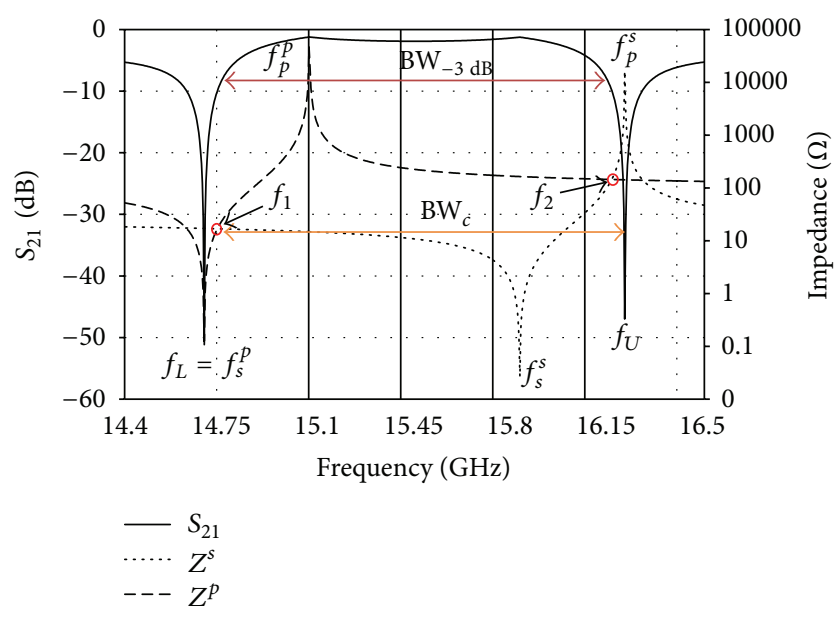

FIGURE 3: Working principle and design parameters of a ladder-type FBAR filter.

frequency, $f_{p}$. At $f_{s}$, the electrical impedance is minimum, $Z_{\text {min }}$, and at $f_{p}$ the electrical impedance is maximum, $Z_{\max }$. At frequency far from $f_{s}$ or $f_{p}$, the impedance has the characteristics of static capacitance, $C_{o}[11]$.

Figure 3 presents the working principle of a second-order ladder-type FBAR filter. The continuous line represents the typical transmission response of ladder filter, while the dotted and dashed lines characterise the electrical impedance of series $\left(Z^{s}\right)$ and parallel $\left(Z^{p}\right)$ resonators, respectively. $f_{s}^{s}$ is the series frequency of the series resonator and $f_{p}^{s}$ is the parallel frequency of the series resonator. Meanwhile, $f_{s}^{p}$ is the series frequency of the shunt resonator and $f_{p}^{p}$ is the parallel frequency of the shunt resonator. The $f_{s}^{p}$ is tuned to be lower than the $f_{s}^{s}$. The ladder-type filter gives a steep rolloff but a poor out-of-band $(\mathrm{OoB})$ rejection characteristic. A better OoB can be achieved by cascading more L-section to the filter; however this will have to be traded off with the insertion loss.

3.1. Filter Design Methodology. Figure 3 described the design parameters of FBAR filter. A set of closed-form expressions for designing FBAR filters are given in [17]. The design of ladder-type FBAR filter consists of determining the order of the filter. The specifications such as bandwidth, OoB rejection, and the frequency allocation of the upper $f_{U}$ and lower $f_{L}$ transmission zeros will be used as design variables. The design procedure is as follows.

Step 1 (determine $f_{s}^{p}$ and $f_{p}^{s}$ ). The value of $f_{s}^{p}$ will be located at the lower transmission zero, $f_{L}$, and $f_{p}^{s}$ will be located at the upper transmission zero, $f_{U}$, as shown in Figure 3.

Step 2 (determine $f_{p}^{p}$ and $f_{s}^{s}$ ). The value of $f_{p}^{p}$ and $f_{s}^{s}$ can be calculated using [14]

$$
k_{\mathrm{eff}}^{2}=\left(\frac{\pi^{2}}{4}\right)\left(\frac{f_{p}-f_{s}}{f_{p}}\right) .
$$

This value can be set as required, but two conditions must be accomplished: the value of $k_{\text {eff }}^{2}$ must be lower than the maximum achievable value with the used materials, and the design is restricted to those filters whose upper and lower transmission zeroes fulfil [17]

$$
f_{U} \geq\left(\frac{\pi^{2}}{4 k_{\mathrm{eff}}^{2}}\right)\left(\frac{\pi-\sqrt{\pi^{2}-16 k_{\mathrm{eff}}^{2}}}{\pi+\sqrt{\pi^{2}-16 k_{\mathrm{eff}}^{2}}}\right) f_{L} .
$$

Step 3 (determine $f_{1}$ and $f_{2}$ ). The approximation of bandwidth $\left(\mathrm{BW}_{c}\right)$ is $\mathrm{BW}_{c}=f_{2}-f_{1}$, where $f_{1}$ and $f_{2}$ are the frequencies where $\left|Z^{s}\left(f_{i}\right)\right|=\left|Z^{p}\left(f_{i}\right)\right|$ and $i=1,2$ is made. The $\mathrm{BW}_{c}$ will be smaller than the $3 \mathrm{~dB}$ bandwidth $\left(\mathrm{BW}_{-3 \mathrm{~dB}}\right)$ and it is independent on the filter order, thus simplifying significantly the design equations.

Step 4 (determine $\psi=C_{o}^{s} / C_{o}^{p}$ ). The $\mathrm{BW}_{c}$ depends on the value of $f_{s}^{p}, f_{p}^{p}, f_{s}^{s}, f_{p}^{s}$, and $\psi$. Since all the resonance frequencies have been determined, the $\psi$ will be calculated to achieve the specified $\mathrm{BW}_{c}$. A simplified approach of electrical impedance of the FBAR is as given in [22]:

$$
Z_{\text {in }}=\frac{1}{j 2 \pi f C_{o}}\left(\frac{f^{2}-f_{s}^{2}}{f^{2}-f_{p}^{2}}\right)
$$

resulting as given in [17]:

$$
\psi=\frac{-f_{i}^{4}+f_{i}^{2} f_{p}^{p 2}+f_{i}^{2} f_{s}^{s 2}-f_{s}^{s 2} f_{p}^{p 2}}{f_{i}^{4}-f_{i}^{2} f_{p}^{s 2}-f_{i}^{2} f_{s}^{p 2}+f_{p}^{s 2} f_{s}^{p 2}},
$$

where $f_{i}$ can be either $f_{1}$ or $f_{2}$. In order to obtain a closedform expression of $\psi$ as a function of $\mathrm{BW}_{c}$, an approximation of $f_{1}$ and $f_{2}$ that are symmetrically spaced around the centre frequency, $f_{c}$, is made, where $f_{1}=f_{c}-\mathrm{BW}_{c} / 2$ and $f_{2}=$ $f_{c}+\mathrm{BW}_{c} / 2$. It can be seen from (4) that $\psi$ increases with $\mathrm{BW}_{c}$.

Step 5 (determine $\Theta=C_{o}^{s} C_{o}^{p}$ ). One of the conditions to find the static capacitance that the filter must accomplish is the image impedance matching condition. This condition is define as [17]

$$
C_{o}^{s} C_{o}^{p}=\frac{1}{\left(2 \pi f_{c} Z_{o}\right)^{2}},
$$

where $Z_{o}=50 \Omega$ is the impedance of the source and the load.

Step 6 (compute $C_{o}^{s}$ and $C_{o}^{p}$ and determine $N$ ). $C_{o}^{s}$ and $C_{o}^{p}$ can now be easily computed using (4) and (5). Since the OoB rejection level depends only on $N$ and $\psi$, the order $N$ of the filter will be chosen to achieve the required $\mathrm{OoB}$ rejection.

In designing a wideband FBAR ladder filter at high frequencies, many considerations are required as discussed in the next section.

\section{Ku-Band FBAR Filter Design}

In this study, the ladder-type FBAR filter is designed to achieve $\mathrm{BW}_{-3 \mathrm{~dB}}$ of $1.2 \mathrm{GHz}$ with centre frequency of 


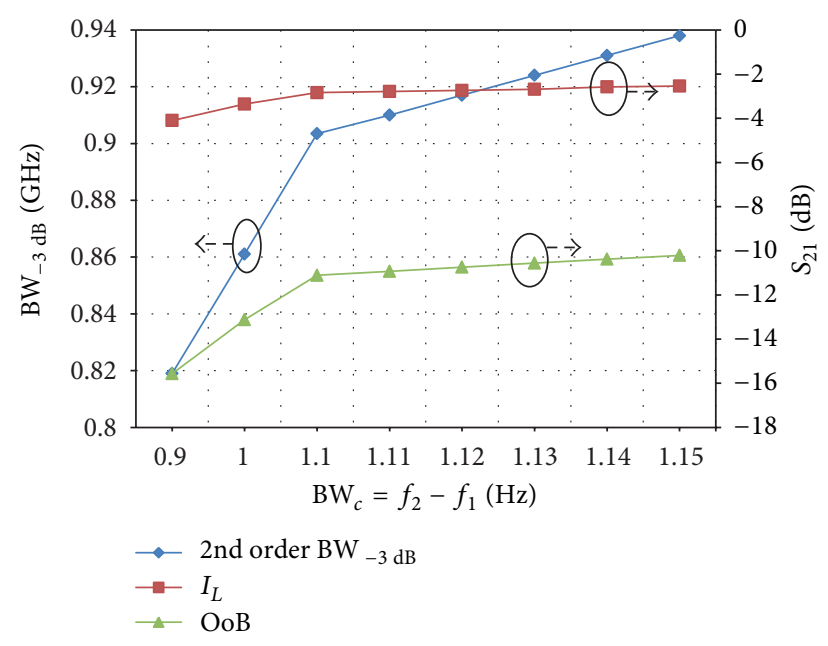

FIGURE 4: Effect of varying the $f_{1}$ and $f_{2}$.

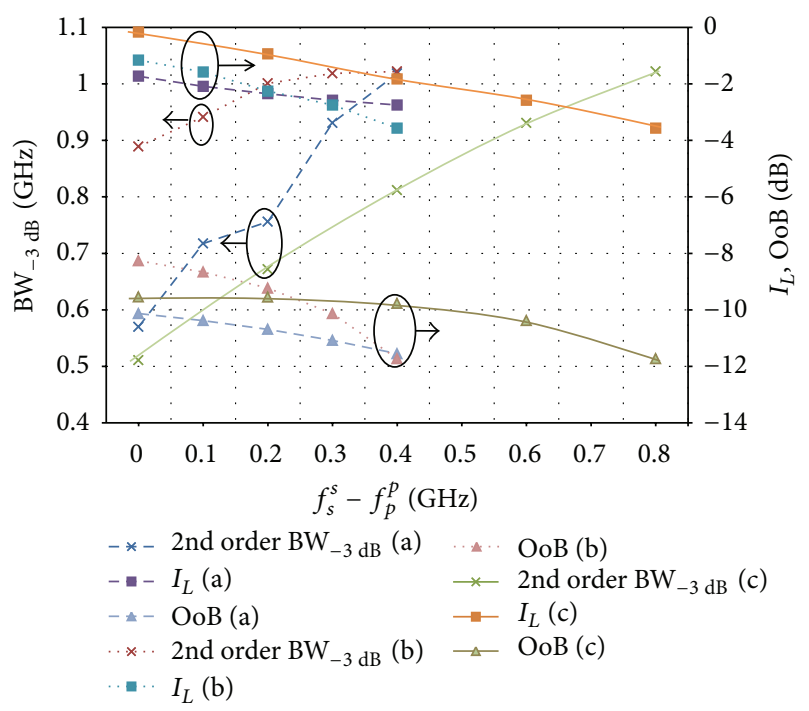

FIGURE 5: Effect of varying $f_{s}^{s}, f_{s}^{p}, f_{p}^{s}$, and $f_{p}^{p}$.

15.5 $\mathrm{GHz}$, insertion loss of $3 \mathrm{~dB}$, and out-of-band rejection of $12 \mathrm{~dB}$. In order to achieve the required specifications of FBAR filter, an analysis of the parameters that affect the characteristics of the filters has been carried out. The effects of varying $f_{1}$ and $f_{2}$ followed by the effect of varying $f_{s}^{s}$, $f_{s}^{p}, f_{p}^{s}$, and $f_{p}^{p}$ are presented in Sections 4.1 and 4.2. Finally, the effects of filter order $N$ on $\mathrm{OoB}$ rejection and filter characteristics are discussed in Section 4.3.

4.1. Effect of $f_{1}$ and $f_{2}$. The effect of $f_{1}$ and $f_{2}$ is shown in Figure 4 , where $\mathrm{BW}_{c}$ is equal to $f_{2}-f_{1}$. Eight different filters are designed to investigate the effect. The bandwidth of each FBAR is set to $500 \mathrm{MHz}$ and the difference between $f_{s}^{s}$ and $f_{p}^{p}$ is set to $600 \mathrm{MHz}$. Second-order filter characteristics are used for comparison. From Figure 4, it is observed that as the $\mathrm{BW}_{c}$ increases, the $\mathrm{BW}_{-3 \mathrm{~dB}}$ also increases. It is also observed that the insertion loss $\left(I_{L}\right)$ has no significant change. Meanwhile

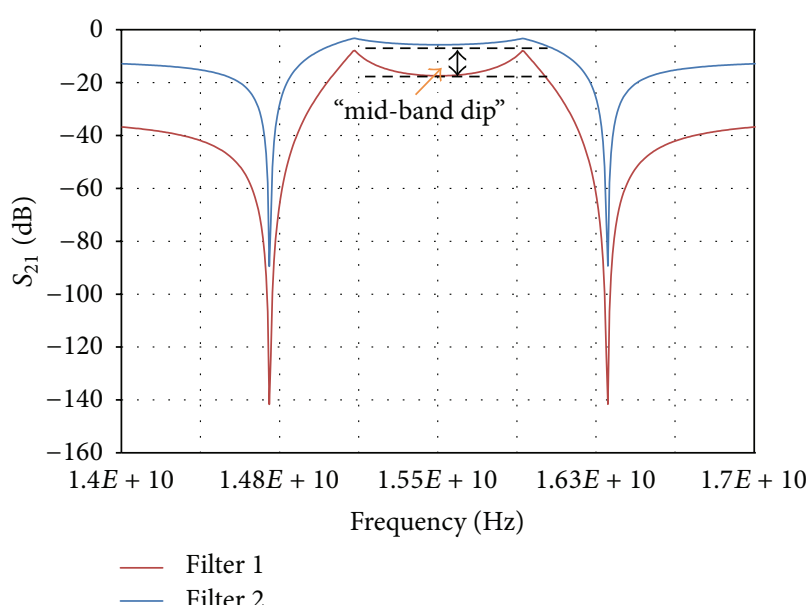

Figure 6: Comparison of transmission response $\left(S_{21}\right)$ of 2 nd order FBAR filter of Filter 1 and Filter 2.

the $\mathrm{OoB}$ rejection decreases significantly after $\mathrm{BW}_{c}$ reaches $1.0 \mathrm{GHz}$ and then remains constant. From this observation, we can conclude that to get wider $\mathrm{BW}_{-3 \mathrm{~dB}}$, the $f_{2}-f_{1}\left(\mathrm{BW}_{c}\right)$ should be increased to the required value.

4.2. Effect of Varying $f_{s}^{s}, f_{s}^{p}, f_{p}^{s}$, and $f_{p}^{p}$. Five different filters are designed to investigate the effect of varying $f_{s}^{s}$ while other parameters are constant and the obtained results are presented as dashed line in Figure 5. Firstly, the value of $f_{p}^{p}$ is kept constant to $15.2 \mathrm{GHz}$ while $f_{s}^{s}$ varies from $15.5 \mathrm{GHz}$ to $15.9 \mathrm{GHz}$ with $100 \mathrm{MHz}$ steps. The results in Figure 5 indicate that as the $f_{s}^{s}-f_{p}^{p}$ decreases, the 2 nd order $\mathrm{BW}_{-3 \mathrm{~dB}}$ also decreases.However, it is observed that the $I_{L}$ and the OoB are inversely proportional to $f_{s}^{s}-f_{p}^{p}$.

Secondly, the value of $f_{s}^{s}$ is kept constant to $15.2 \mathrm{GHz}$, while $f_{p}^{p}$ varies from $15.5 \mathrm{GHz}$ to $15.9 \mathrm{GHz}$ with $100 \mathrm{MHz}$ steps. The results achieved are depicted as dotted line in Figure 5. It can be seen that as the $f_{s}^{s}-f_{p}^{p}$ decreases, the $\mathrm{BW}_{-3 \mathrm{~dB}}$ decreases. It is also observed that $I_{L}$ improves, while the $\mathrm{OoB}$ degrades as the $\mathrm{BW}_{-3 \mathrm{~dB}}$ decreases.

The effect of varying both $f_{s}^{s}$ and $f_{p}^{p}$ while other parameters are constant is shown as continuous line in Figure 5. It shows that as the $f_{s}^{s}-f_{p}^{p}$ decreases $\mathrm{BW}_{-3 \mathrm{~dB}}$ also decreases. $I_{L}$ improves significantly as $\mathrm{BW}_{-3 \mathrm{~dB}}$ decreases. On the other hand, the $\mathrm{OoB}$ degrades as $\mathrm{BW}_{-3 \mathrm{~dB}}$ decreases.

Based on the analysis described in this section, varying the values of $f_{s}^{s}, f_{s}^{p}, f_{p}^{s}$, and $f_{p}^{p}$ will directly affect the filter characteristics. From Figure 5, it is observed that the BW $-3 \mathrm{~dB}$ and $\mathrm{OoB}$ gradually decrease as the difference between $f_{s}^{s}$ and $f_{p}^{p}$ decreases. On the other hand, $I_{L}$ improves as $f_{s}^{s}$ and $f_{p}^{p}$ decrease.

Figure 6 presents the comparison of transmission response $\left(S_{21}\right)$ of the filters designed in this analysis. It is observed that some of the filters have the "mid-band dip" at the centre due to the increasing gap between $f_{s}^{s}$ and $f_{p}^{p}$ of the FBARs. Hence, one of the purposes of this analysis is to minimise the "mid-band dip." Both filters have same values 
TABLE 2: FBAR filters design parameters.

\begin{tabular}{lcccccccc}
\hline Parameter & $f_{1}(\mathrm{GHz})$ & $f_{2}(\mathrm{GHz})$ & $f_{s}^{s}(\mathrm{GHz})$ & $f_{p}^{s}(\mathrm{GHz})$ & $f_{s}^{p}(\mathrm{GHz})$ & $f_{p}^{p}(\mathrm{GHz})$ & $\mathrm{BW}_{c}(\mathrm{GHz})$ & $\psi$ \\
\hline Filter A & 14.725 & 16.275 & 15.90 & 16.30 & 14.70 & 15.10 & 1.55 & 5.2 \\
Filter B & 14.75 & 16.25 & 15.90 & 16.30 & 14.70 & 15.10 & 1.50 & 11.2 \\
\hline
\end{tabular}

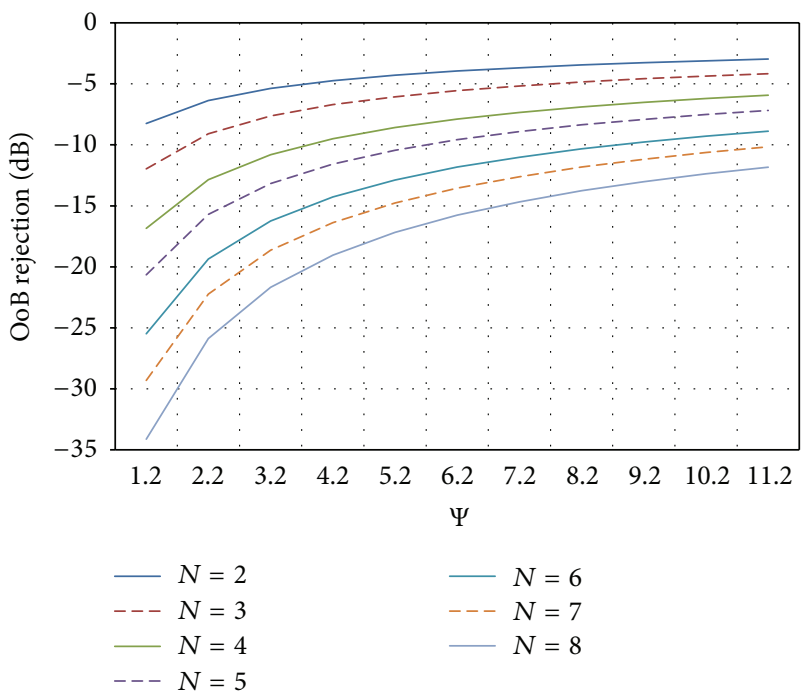

FIgURE 7: Out-of-band rejection $(\mathrm{OoB})$ as a function of $\Psi$ and $N$.

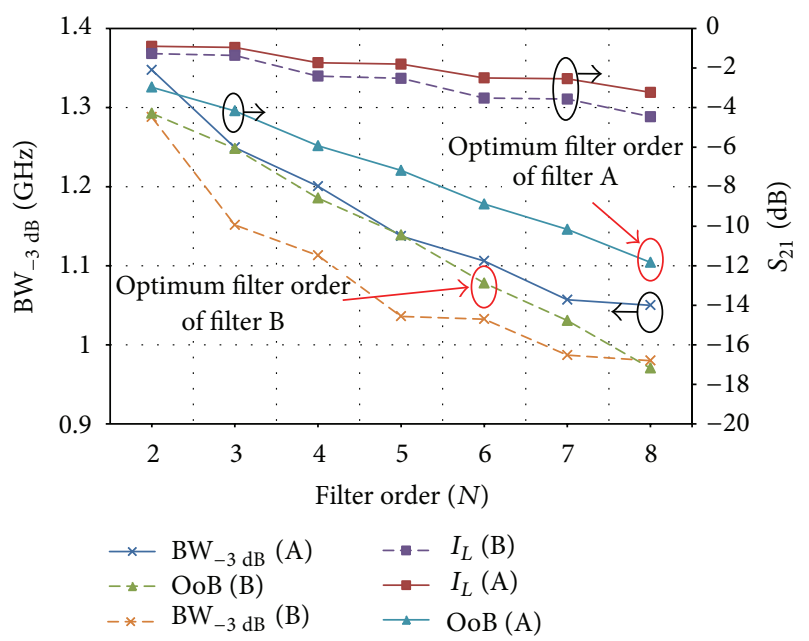

FIGURE 8: Characteristics of Ku-band FBAR filters.

of $f_{s}^{s}, f_{s}^{p}, f_{p}^{s}$, and $f_{p}^{p}$ but different values of $f_{1}$ and $f_{2}$. As mentioned previously, $\mathrm{BW}_{c}=f_{2}-f_{1}$. Filter 2 has the $\mathrm{BW}_{c}$ of $1.55 \mathrm{GHz}$ while Filter 1 has the $\mathrm{BW}_{c}$ of $1.14 \mathrm{GHz}$. It can be seen that for Filter 2 the "mid-band dip" is less than $3 \mathrm{~dB}$ and the achieved $\mathrm{BW}_{-3 \mathrm{~dB}}$ for these filters is $1.0 \mathrm{GHz}$. The variation of $f_{1}$ and $f_{2}$ values has significant effect on $\psi$. The relationship of $\mathrm{OoB}, \psi$, and $N$ depicted in Figure 7. From Figure 7 it can be observed that an increase in $\psi$ will degrade the OoB. As a result of this analysis, the FBAR filter design parameters listed in Table 2 have been chosen.

Next we will discuss optimisation of filter order for these filters.
4.3. Effect of $N$. The OoB of the FBAR filter only depends on $\psi$ and $N$. As $\psi$ is already determined previously, the $N$ is chosen to achieve the specified $\mathrm{BW}_{-3 \mathrm{~dB}}$. The effect of $N$ on filter characteristics of Filters A and B is presented in Figure 8. It is noticed that the $\mathrm{BW}_{-3 \mathrm{~dB}}$ and $I_{L}$ decrease gradually as $N$ increases. At the same time OoB steadily improves as $N$ increases. From this study, it is noticeably seen that even though increasing the filter order will improve the OoB, it will degrade $I_{L}$ and $\mathrm{BW}_{-3 \mathrm{~dB}}$ of the filter. The higher filter order requires more resonators which will result in increased size and cost. Hence, optimum value of filter order is selected to be 8 th and 5 th for filter $A$ and filter $\mathrm{B}$, respectively.

\section{Results}

From the analysis described above, it is possible to design FBAR filters with $1.0 \mathrm{GHz}$ bandwidth operating at $15.5 \mathrm{GHz}$. The parameters used in designing the filter are presented in Table 2. The characteristics of each filter are summarised and compared in Figure 8. It is observed that Filter A has the advantage of wider $\mathrm{BW}_{-3 \mathrm{~dB}}$ and better $I_{L}$ compared to Filter B. However, the drawback of Filter $A$ is the OoB rejection which is slightly higher than that of Filter B. Filter A has achieved the $\mathrm{BW}_{-3 \mathrm{~dB}}$ up to $1.35 \mathrm{GHz}$ and extensive value of insertion loss, but the OoB rejection is too high. From all the analysis, it can be seen that FBAR filter with wider $\mathrm{BW}_{-3 \mathrm{~dB}}$ will degrade the $\mathrm{OoB}$ rejection of the filter, even though the $I_{L}$ is improved.

Table 3 shows a comparison of FBAR filters operating in $\mathrm{X}$-band to Ka-band. All these filters were designed at 4-stage ladder filter which is equal to 8th order filter. The 7th and 8th order FBAR filter A and the 5th and 6th order FBAR filter B are chosen as the best filter characteristics in this work. It is shown that FBAR filters in this work have better performance than reported in [14], where the insertion loss is too high and the fractional bandwidth is only $2 \%$. To date, this is the first FBAR filter operating in $\mathrm{Ku}$-band frequency range ever reported.

Based on the characteristics of the designed FBAR filters, two sets of FBARs were designed using the BVD equivalent circuit. The derived equations for equivalent circuit elements of FBAR are as presented in [23]. The FBAR parameters extracted are listed in Table 4.

In order to further analyse and optimise the FBAR designs evaluated using numerical analysis, 3D Finite Element Method (FEM) was used. Tables 5 and 6 show the comparisons of the extracted FBAR parameters between the 1-D and 3-D FEM analyses for Filter A and Filter B. It can be seen that the parameter values are highly similar.

Figure 9 shows the transmission response $\left(S_{21}\right)$ of the 3rd stage FBAR filter A designed using the 1-D modelling and 3-D FEM. The results from both analyses match well 
TABLE 3: Comparison of FBAR filter characteristics.

\begin{tabular}{lcccc}
\hline Reference & Centre frequency $(\mathrm{GHz})$ & Insertion loss $(\mathrm{dB})$ & Out-of-band $(\mathrm{GHz})$ & Fractional bandwidth $(\%)$ \\
\hline$[13]$ & 29.2 & 3.8 & 11 & 3.4 \\
& 23.8 & 3.8 & 13 & 3.4 \\
{$[14]$} & 19.8 & 4.1 & 18 & 2.0 \\
{$[15]$} & 9.08 & 1.7 & 12 & 3.1 \\
Filter A $^{*}$ & 15.5 & 3.2 & 13 & 6.45 \\
Filter B $^{*}$ & 15.5 & 3.5 & 6.45 \\
\hline
\end{tabular}

*indicates the FBAR filter characteristics in this work.

TABLE 4: Extracted FBAR parameters.

\begin{tabular}{lcccc}
\hline Parameter & \multicolumn{2}{c}{ Filter A } & \multicolumn{2}{c}{ Filter B } \\
FBAR type & $\begin{array}{l}\text { FBAR 1 } \\
\text { (series) }\end{array}$ & $\begin{array}{c}\text { FBAR 2 } \\
\text { (shunt) }\end{array}$ & $\begin{array}{c}\text { FBAR 1 } \\
\text { (series) }\end{array}$ & $\begin{array}{c}\text { FBAR 2 } \\
\text { (shunt) }\end{array}$ \\
\hline$C_{o}(\mathrm{pF})$ & 0.6872 & 0.0614 & 0.4681 & 0.0901 \\
$C_{m}(\mathrm{pF})$ & 0.0350 & 0.0379 & 0.0239 & 0.0258 \\
$L_{m}(\mathrm{nH})$ & 2.8613 & 34.616 & 4.2 & 23.582 \\
$k_{\mathrm{eff}}^{2}(\%)$ & 6.06 & 6.54 & 6.06 & 6.54 \\
$f_{s}(\mathrm{GHz})$ & 15.904 & 14.707 & 15.904 & 14.707 \\
$f_{p}(\mathrm{GHz})$ & 16.313 & 15.12 & 16.313 & 15.12 \\
\hline
\end{tabular}

TABLE 5: Comparisons of 1D and 3D FEM of Filter A.

\begin{tabular}{lcccc}
\hline \multirow{2}{*}{ Parameters } & \multicolumn{2}{c}{ Series FBAR } & \multicolumn{2}{c}{ Shunt FBAR } \\
& 1D & 3D FEM & 1D & 3D FEM \\
\hline$C_{o}(\mathrm{pF})$ & 0.6872 & 0.6837 & 0.0614 & 0.0651 \\
$C_{m}(\mathrm{pF})$ & 0.0351 & 0.0373 & 0.0379 & 0.0357 \\
$L_{m}(\mathrm{nH})$ & 2.86 & 2.69 & 34.616 & 32.897 \\
$k_{\mathrm{eff}}^{2}(\%)$ & 6.06 & 6.36 & 6.54 & 6.51 \\
$f_{s}(\mathrm{GHz})$ & 15.90 & 15.87 & 14.70 & 14.67 \\
$f_{p}(\mathrm{GHz})$ & 16.30 & 16.30 & 15.10 & 15.12 \\
\hline
\end{tabular}

TABLE 6: Comparisons of 1D and 3D FEM of Filter B.

\begin{tabular}{lcccc}
\hline \multirow{2}{*}{ Parameters } & \multicolumn{2}{c}{ Series FBAR } & \multicolumn{2}{c}{ Shunt FBAR } \\
& $1 \mathrm{D}$ & 3D FEM & 1D & 3D FEM \\
\hline$C_{o}(\mathrm{pF})$ & 0.468 & 0.471 & 0.0901 & 0.107 \\
$C_{m}(\mathrm{pF})$ & 0.024 & 0.025 & 0.058 & 0.058 \\
$L_{m}(\mathrm{nH})$ & 4.20 & 3.97 & 23.58 & 19.96 \\
$k_{\mathrm{eff}}^{2}(\%)$ & 6.06 & 6.36 & 6.54 & 6.51 \\
$f_{s}(\mathrm{GHz})$ & 15.90 & 15.87 & 14.70 & 14.67 \\
$f_{p}(\mathrm{GHz})$ & 16.30 & 16.29 & 15.10 & 15.12 \\
\hline
\end{tabular}

within the pass-band. The attenuation poles of both filters also agree well. The disagreement on the suppression is due to piezoelectric material losses that were introduced in 3-D FEM. It can be concluded that the 1-D modelling used can be applied to predict the characteristics of the FBAR filter.

\section{Conclusion}

FBAR filter with insertion loss of $3.5 \mathrm{~dB}$, out-of-band rejection of $12 \mathrm{~dB}$, centre frequency of $15.5 \mathrm{GHz}$, and fractional

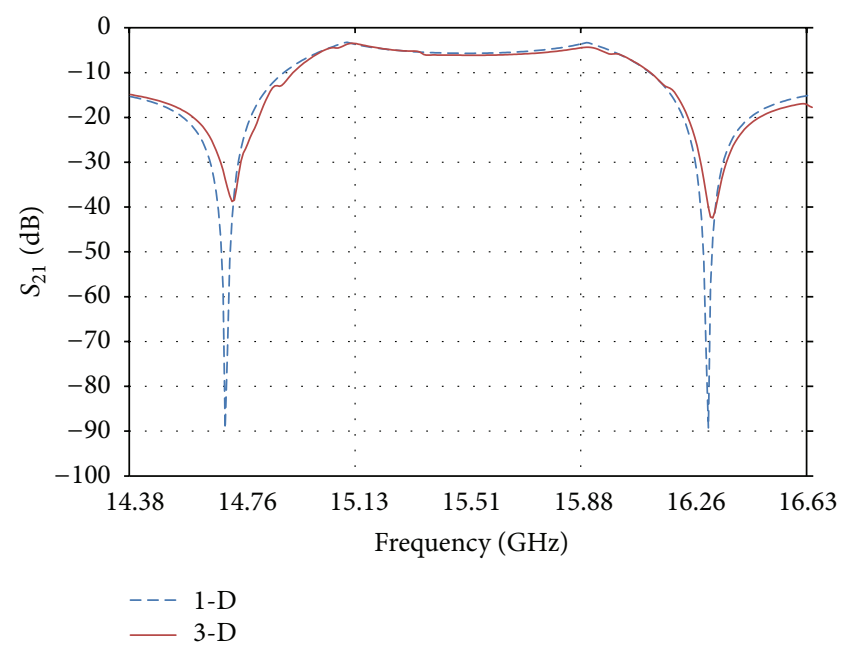

FIgURE 9: Comparison of FBAR filter transmission response $\left(S_{21}\right)$ between $1 \mathrm{D}$ and 3D FEM.

bandwidth of 6.45\% was designed using closed-form expressions. To best of the author's knowledge this analysis has for the first time shown that the closed-form expressions and 1$D$ models can be used to characterise wideband FBAR filters. From the analysis, we conclude that increasing the order of the FBAR filter will improve the out-of-band rejection; however the insertion loss and the $3 \mathrm{~dB}$ bandwidth will degrade. The results show that the proposed FBAR filter is a good candidate for implementation in a Ku-band transceiver.

\section{References}

[1] M. F. Islam, M. A. Mohd. Ali, and B. Y. Majlis, "Ku-band bandpass filter using surface micromachined process," in Proceedings of the Asia Pacific Microwave Conference (APMC '09), pp. 547550, December 2009.

[2] L. Chang-Ho, A. Sutono, H. Sangwoo et al., "A compact LTCCbased Ku-band transmitter module," IEEE Transactions on Advanced Packaging, vol. 25, no. 3, pp. 374-384, 2002.

[3] H. Su, M. Xing, Y. Li, and W. Li, "Design of a front-end of a Ku-band transceiver based on LTCC technology," in Proceedings of the 11th International Conference on Electronic Packaging Technology and High Density Packaging (ICEPT-HDP '10), pp. 851-855, August 2010.

[4] D. Kim, D. H. Kim, G. H. Baek et al., "An FBAR-and LTCCbased RF front-end module for Wi-Fi and WiMAX dual-mode 
systems," Microwave and Optical Technology Letters, vol. 52, no. 3, pp. 753-757, 2010.

[5] G. G. Fattinger, J. Kaitila, R. Aigner, and W. Nessler, "Thin film bulk acoustic wave devices for applications at $5.2 \mathrm{GHz}$," in Proceedings of the IEEE Ultrasonics Symposium, pp. 174-177, October 2003.

[6] H. C. Pineda, Thin Film bilk acoustic wave resonator-FBAR fabrication, heterogeneous integration with CMOS technologies, and sensor applications [Ph.D. thesis], Universite de Montpellier II, Montpellier, France; Universitat Autonoma de Barcelona, Barcelona,Spain, 2007.

[7] K.-W. Kim, G.-Y. Kim, J.-G. Yook, and H.-K. Park, "Air-gaptype TFBAR-based filter topologies," Microwave and Optical Technology Letters, vol. 34, no. 5, pp. 386-387, 2002.

[8] K. Umeda, H. Kawamura, M. Takeuchi, and Y. Yoshino, "Characteristics of an AlN-based bulk acoustic wave resonator in the super high frequency range," Vacuum, vol. 83, no. 3, pp. 672674, 2008.

[9] M. Hara, T. Yokoyama, T. Sakashita et al., "Super-highfrequency band filters configured with air-gap-type thin-film bulk acoustic resonators," Japanese Journal of Applied Physics, vol. 49, no. 7, Article ID 07HD13, 2010.

[10] N. I. M. Nor, K. Shah, J. Singh, N. Khalid, and Z. Sauli, "Design and analysis of film bulk acoustic wave resonator in Ku-band frequency for wireless communication," in Proceeding of SPIE, Active and Passive Smart Structures and Integrated Systems 2012, 83411R, vol. 8341, 2012.

[11] F. Z. Bi and B. P. Barber, "Bulk acoustic wave RF technology," IEEE Microwave Magazine, vol. 9, no. 5, pp. 65-80, 2008.

[12] A. A. Shirakawa, J.-M. Pham, P. Jarry, and E. Kerhervé, "Design of FBAR filters at high frequency bands," International Journal of RF and Microwave Computer-Aided Engineering, vol. 17, no. 1, pp. 115-122, 2007.

[13] M. Hara, T. Yokoyama, T. Sakashita, M. Ueda, and Y. Satoh, "A study of the thin film bulk acoustic resonator filters in several ten $\mathrm{GHz}$ band," in Proceedings of the IEEE International Ultrasonics Symposium (IUS '09), pp. 851-854, September 2009.

[14] M. Hara, T. Yokoyama, M. Ueda, and Y. Satoh, "X-band filters utilizing AlN thin film bulk acoustic resonators," in Proceedings of the IEEE Ultrasonics Symposium (IUS '07), pp. 1152-1155, October 2007.

[15] T. Yokoyama, M. Hara, M. Ueda, and Y. Satoh, "K-band ladder filters employing air-gap type thin film bulk acoustic resonators," in Proceedings of the IEEE International Ultrasonics Symposium (IUS '08), pp. 598-601, November 2008.

[16] G. F. Perez-Sanchez and A. Morales-Acevedo, "Design of bulk acoustic wave resonators based on $\mathrm{ZnO}$ for filter applications," in Proceedings of the 6th International Conference on Electrical Engineering, Computing Science and Automatic Control (CCE '09), pp. 1-6, November 2009.

[17] O. Menéndez, P. De Paco, R. Villarino, and J. Parrón, "Closedform expressions for the design of ladder-type FBAR filters," IEEE Microwave and Wireless Components Letters, vol. 16, no. 12, pp. 657-659, 2006.

[18] C. Min-Chiang, H. Zi-Neng, P. Shih-Yung, Z. Wang, and C. S. Lam, "Modified BVD-equivalent circuit of FBAR by taking electrodes into account," in Proceedings of the IEEE Symposium on Ultrasonics, pp. 973-976, October 2002.

[19] J. Jianhua, L. Yinqiao, H. Sha, J. Zhou, Y. Fei, and F. Tan, "A compact LTCC Transmit Receive module at Ku-band," in Proceedings of the International Conference on Microwave and
Millimeter Wave Technology (ICMMT '10), pp. 1239-1241, May 2010.

[20] R. Gilmore, C. Bruckner, and D. Dunn, "Ku-band MMIC transceiver for mobile satellite communications," in Proceedings of the 21st European Microwave Conference, pp. 1261-1265, September 1991.

[21] Z. Zhang, G. Zhao, X. Zeng, H. Sun, and X. Lv, "A transceiver for Ku band digital monopulse radar system," in Proceedings of the International Conference on Microwave and Millimeter Wave Technology (ICMMT '10), pp. 979-982, May 2010.

[22] A. Shirakawa, J. M. Pham, P. Jarry, E. Kerherve, and E. Hanna, "Ladder-type FBAR filter synthesis methodology," in Proceedings of the 19th Conference on Design of Circuits and Integrated Systems (DCIS '04), pp. 519-523.

[23] K. Kun-Wook, G. Myeong-Gweon, Y. Jong-Gwan, and P. Han$\mathrm{Kyu}$, "Resonator size effects on the TFBAR ladder filter performance," IEEE Microwave and Wireless Components Letters, vol. 13, no. 8, pp. 335-337, 2003. 

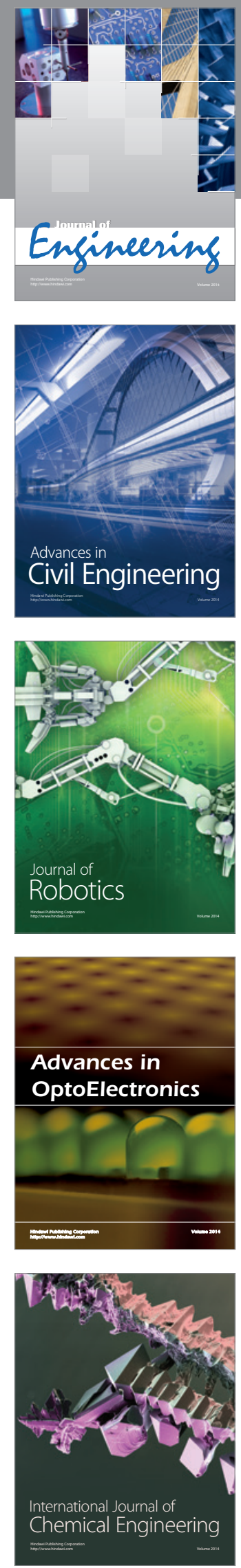

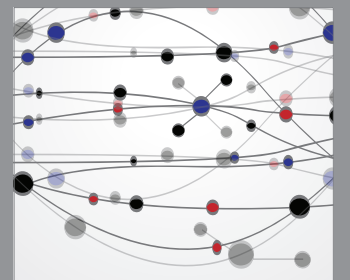

The Scientific World Journal
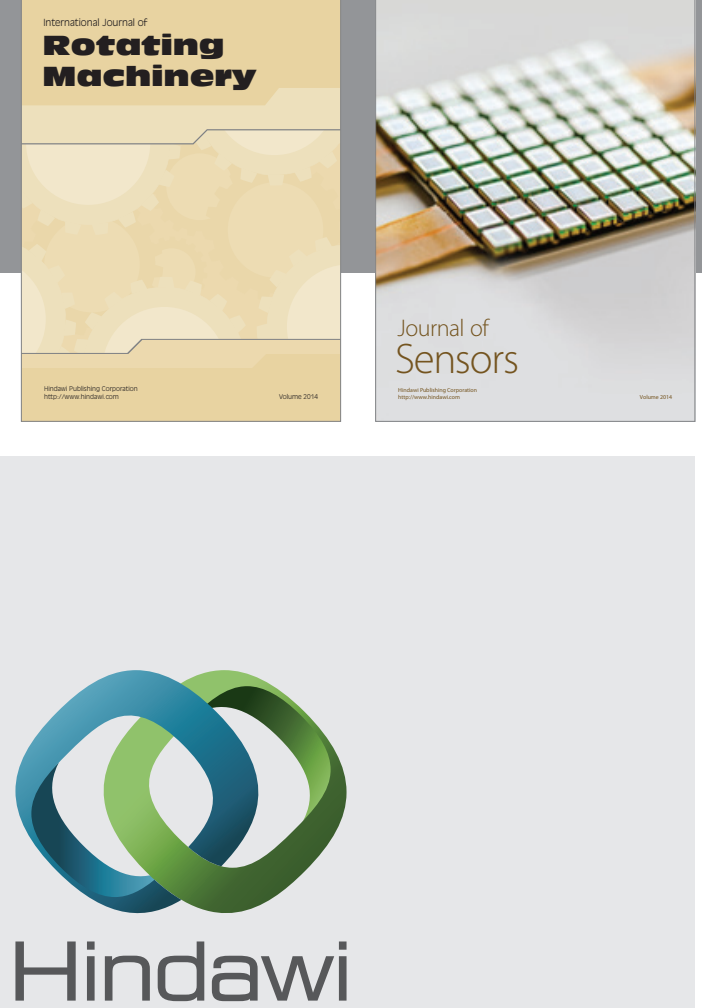

Submit your manuscripts at http://www.hindawi.com
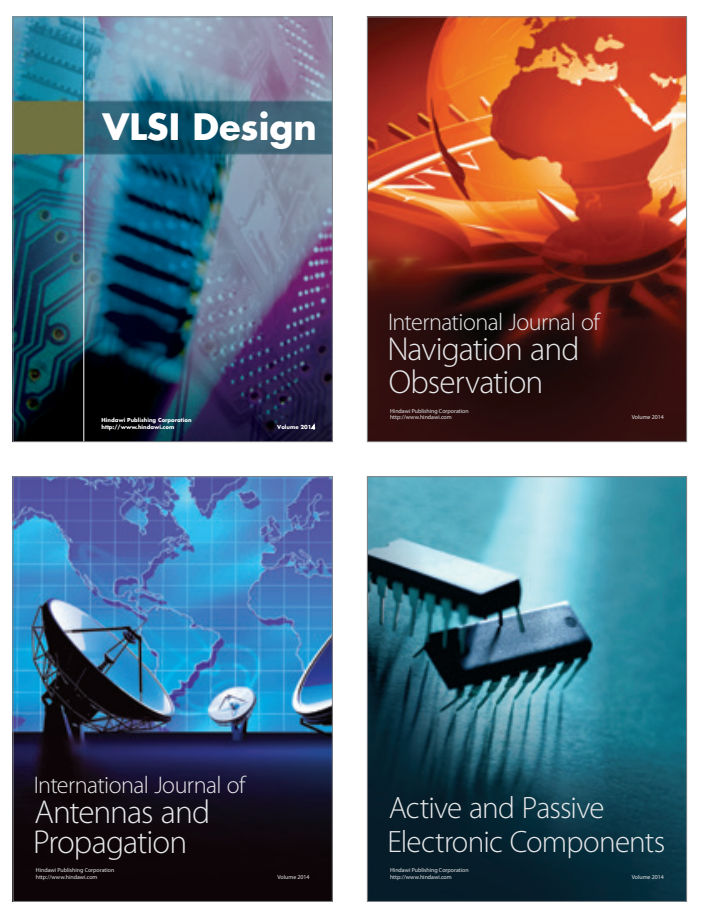
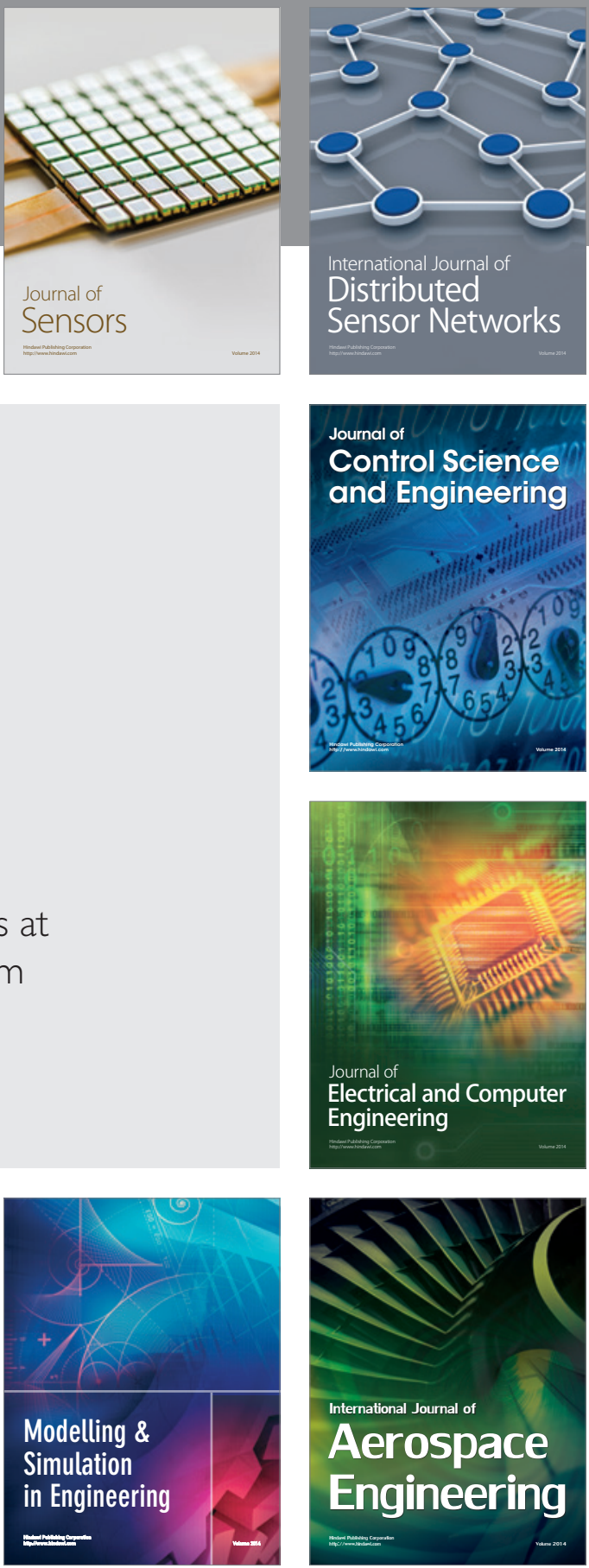

Journal of

Control Science

and Engineering
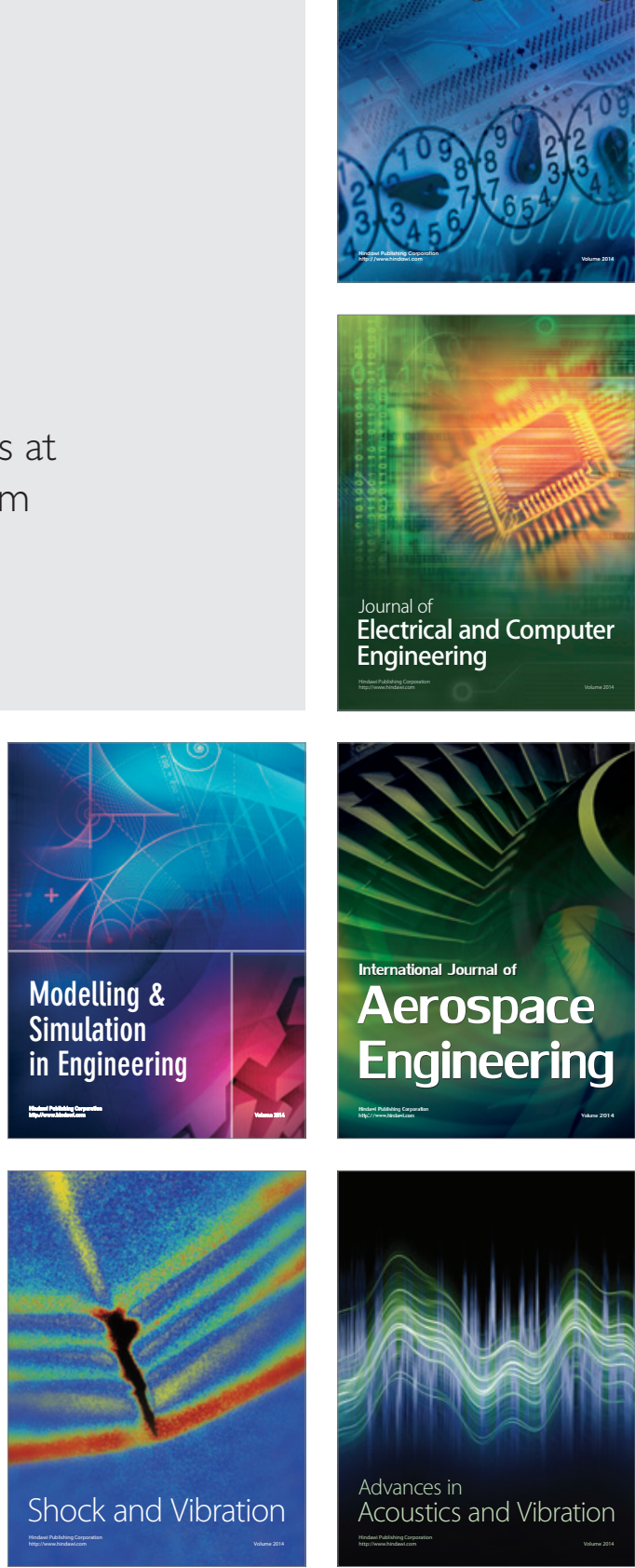